\title{
Analysis of Bifurcation Behavior of a Piecewise Linear Vibrator with Electromagnetic Coupling for Energy Harvesting Applications
}

\author{
A. EL AROUDI* \\ Department of Electronics, Electrical Engineering and Automatic Control \\ Universitat Rovira i Virgili URV, Tarragona, Spain, \\ Email: abdelali.elaroudi@urv.cat \\ H. OUAKAD \\ Mechanical Engineering Department, \\ King Fahd University of Petroleum and Minerals, \\ Dhahran, PO Box. 31261, Kingdom of Saudi Arabia, \\ Email: houakad@kfupm.edu.sa \\ L. BENADERO \\ Departament de Física Aplicada, \\ Universitat Politècnica de Catalunya UPC, Barcelona, Spain, \\ Email:luis@fa.upc.edu \\ M. YOUNIS \\ Physical Sciences and Engineering Division, \\ King Abdullah University of Science and Technology KAUST, \\ Kingdom of Saudi Arabia, \\ Email:mohammad.younis@kaust.edu.sa
}

Received (to be inserted by publisher)

\begin{abstract}
Recently, nonlinearities have been shown to play an important role in increasing the extracted energy of vibration-based energy harvesting systems. In this paper, we study the dynamical behavior of a piecewise linear (PWL) spring-mass-damper system for vibration-based energy harvesting applications. First, we present a continuous time single degree of freedom PWL dynamical model of the system. Different configurations of the PWL model and their corresponding state-space regions are derived. Then, from this PWL model, extensive numerical simulations are carried out by computing time-domain waveforms, state-space trajectories and frequency responses under a deterministic harmonic excitation for different sets of system parameter values. Stability analysis is performed using Floquet theory combined with Fillipov method, Poincaré map modeling and finite difference method (FDM). The Floquet multipliers are calculated using these three approaches and a good concordance is obtained among them. The performance of the system in terms of the harvested energy is studied by considering both purely harmonic excitation and a noisy vibrational source. A frequency-domain analysis shows that the harvested energy could be larger at low frequencies as compared to an equivalent linear system, in particular for relatively low excitation intensities. This could be an advantage for potential use of this system in low frequency ambient vibrational-based energy harvesting applications.
\end{abstract}

*Author for correspondence abdelali.elaroudi@urv.cat 
Keywords: Nonlinear Energy Harvester, Stability Analysis, Bifurcations, Poincaré map, Fillipov Method, Floquet Theory, Finite Difference Method.

\section{Introduction}

Recently, there has been an increasing interest in self-powered devices in remote environment applications, where the use of rechargeable batteries becomes inviable or problematic, such as in hard-to-access locations. Some approaches have been investigated in the literature to solve this problem by using energy harvesting techniques [Kaźmierski \& Beeby, 2011]. This topic is considered one of the key points in the development of autonomous sensors with extended lifetime [Mitcheson et al., 2008]. There exist different energy harvesting technologies that can be used depending on the type of the available energy. Among the commonly used energy sources, vibrational kinetic energy is the most used for applications such as development of micro generators and noise harvesters [Kaźmierski \& Beeby, 2011]. In this approach, mechanical energy is converted to electricity by using piezoelectric, capacitive or inductive transducers [Kaźmierski \& Beeby, 2011]. In the piezoelectric transduction the dynamical strain is converted into electrical energy, in inductive conversion, oscillation of magnets induces electric current in coils while in capacitive transduction, geometrical variations induce voltage. Vibration-based energy harvesting research has largely focused on linear electromechanical devices excited at resonance [Kaźmierski \& Beeby, 2011]. In most of the reported studies, the energy harvesters are designed as linear resonators by matching the resonant frequency of the harvester with that of the external excitation to extract maximum power. This maximum power extraction depends on the quality factor ( $Q$ factor) of the linear resonator. However, it is viable only when the excitation frequency is known a priori. Moreover, a maximum energy extraction with a high $\mathrm{Q}$ factor will paradoxically imply a limited and narrow frequency range within which energy can be harvested. The performance of these systems is therefore rapidly degraded if the excitation frequency is far from the resonant one and they are efficient effective when an optimum design is implemented by tuning the resonance frequency to match with the ambient source vibrations frequency. However, in environments where no single dominant frequency exists, these performances can be lowered significantly as the excitation frequency moves away from the designed frequency [Gammaitoni et al., 2011]. Some solutions have been reported recently to remedy these problems. Among them, resonance tuning and frequency up-conversion techniques [Tang et al., 2000; Ralman et al., 2010]. These methods can overcome the above mentioned problems at the expense of making their implementation a challenging task. For instance, resonance tuning implies the change of the mass of the harvester while frequency-up conversion would imply the use of an array of resonators which would increase the size and cost of the harvester and making it not a suitable choice for small self-powered portable devices. Traditionally, nonlinearities are to be avoided in device design. However, recently these nonlinearities have been shown to have potential to allow designers to take advantages of nonlinear behavior in certain applications [Gammaitoni et al., 2011] where the performance of energy harvesters is enhanced by inducing a bistable potential well through introducing suitable polynomial nonlinearities inducing a double well potential effect which makes the harvester efficient in a broad frequency range including low frequencies. By using this approach, rather than resonance frequency tuning, the nonlinearity of the system is exploited to improve the performances of the energy harvester within a wide frequency range outperforming, in this way, classical resonant energy harvesters [Gammaitoni et al., 2011], [Trigona et al., 2011]. In [Andò et al., 2010] another double well working principle was proposed, modeled and numerically simulated using statistical differential equations approach by considering a random input vibration source to describe the behavior of micro-electromechanical systems (MEMS). In [Masana \& Daqqaq, 2012], a methodology for nonlinear energy harvesting by exploiting super-harmonic resonances of a bistable vibrator was proposed lowering, in this way, the bandwidth to low frequency ranges. It has been demonstrated both theoretically and experimentally that a harvester with a bistable potential energy well can exhibit large voltage levels that are favorable for energy harvesting at frequencies close to one half of the excitation frequency. These techniques have been demonstrated to work both at the micro scale [Gammaitoni et al., 2009] and nano scale [López-Suárez et al., 2011]. Polynomial nonlinearity is not the only way to enhance the performances of the harvesters at low frequencies. In [Triplett et al., 2011] a piecewise linear (PWL) system was proposed for vibration-based energy harvesting applications. The performance of the harvester when some suitable parameters vary were characterized by numerical simulations. In [El Aroudi et al., 2013a], this system was reconsidered and its dynamical behavior was studied by 
time-domain and frequency-domain numerical simulations using long-time integration performed on a continuoustime PWL model. Other studies dealing with PWL systems that can be used as energy harvesters can be found in [Bendame and Abdel-Rahman, 2012], [Soliman et al., 2008] where a prototype of an electromagnetic micro generator is designed and analyzed both numerically and experimentally. PWL switched systems constitute a special class of hybrid systems [Liberzon, 2003] and arise often in practical control systems when some nonlinear components such as switching, dead-zone, saturation, relays, and hysteresis are encountered. Most of the PWL systems studied in the literature are characterized by switching among linear subsystems when certain fixed boundaries in the state space are reached [Mandal et al., 2013-a], [Mandal et al., 2013-b], [Xiong et al., 2013], [Xie et al., 2013]. Although each subsystem is linear, the dynamics of the overall switching system is highly nonlinear and its analysis requires sophisticated computational tools [Mandal and Banerjee, 2012].

The aim of this paper is to present some analytical tools to study the nonlinear behavior of this kind of systems and in particular to apply these tools to study in details the dynamical behavior and to carry out the stability analysis of the system considered recently in [Triplett et al., 2011] and [El Aroudi et al., 2013a]. Different approaches will be combined to reveal the richness of the dynamical behaviors that this system can exhibit and to perform its stability analysis. For such a switched complex system, cross checking the results using different tools is necessary. In this paper, stability analysis is performed using Floquet theory combined with Fillipov technique, Poincaré map modeling and Finite Difference Method (FDM). Discrete-time Poincaré map approach allows one to obtain the fixed point corresponding to the steady-state periodic orbit in the continuous domain. By performing a small-signal perturbation in the vicinity of this fixed point, the Jacobian matrix of the discrete time-model is obtained and stability analysis can be carried out straightforwardly. Although by using Floquet theory combined with Fillipov method, the stability analysis can be carried out by using the monodromy matrix in the same way, there is a still a need to use the discrete-time modeling to get the steady-state periodic orbit at which the monodromy matrix must be evaluated. In both previous approaches, the switching sequence of the periodic orbit must be determined beforehand. This is not the case of the FDM in which the switching sequence is not needed.

The rest of the paper is organized as follows. In Section 2, a brief description of the PWL system considered in this study is provided. The dynamical model of this system is presented and its different configurations are unfolded in Section 3. In Section 4, stability analysis is performed using Floquet theory combined with Fillipov technique, Poincaré map modeling and FDM. Numerical simulations illustrating bifurcation diagrams, time-domain waveforms and state-space trajectories are presented in Section 5 when the some suitable system parameters are varied. The Floquet multipliers are calculated for different periodic orbits using different approaches all giving the same result. Symmetry breaking or pitchfork, flip or period doubling and chaotic motion are shown to occur in the system. The performance of the system in terms of the harvested energy is studied in the same section. Finally, conclusions and discussions are presented in the last section.

\section{System description}

Vibration-based energy harvesting is the process in which mechanical energy is transformed into electricity. This process allows to convert the kinetic energy from a moving body due to ambient vibrations into electrical energy through a certain electromechanical mechanism. Energy harvesters can be modeled by single degree of freedom spring-mass systems. In the present work we study the dynamical behavior of a PWL energy harvester first considered in [Triplett et al., 2011]. The schematic diagram of the system is shown in Fig. 1. It consists of a spring-mass-damper system where a piecewise linear (PWL) model for the friction and the stiffness is considered which represents their respective spatial limitation [Triplett et al., 2011]. The total damping of the system is composed of a constant mechanical damping $b_{m}$ and an electromagnetic damping $b_{e}$ described as [Soliman et al., 2008], [Tulloch, 2011]

$$
b_{e}=\frac{(B \ell)^{2}}{R_{C}+R_{L}}
$$

where $B$ is the induced magnetic field, $\ell$ is the length of the coil, $R_{C}$ is its resistance and $R_{L}$ is the load resistance. The piecewise linearity comes from the fact that the electromechanical coupling is assumed to be significant only over a finite interval for the displacement $x$ of the mass $m$. Moreover, the restoring force $f_{r}(x)$ acting on the inertial mass is assumed to contain an interval of zero stiffness, with a linear term outside this region. In particular, the state dependent mechanical damping factor $\beta(x)$ can be expressed as the sum of an electromechanical damping term 


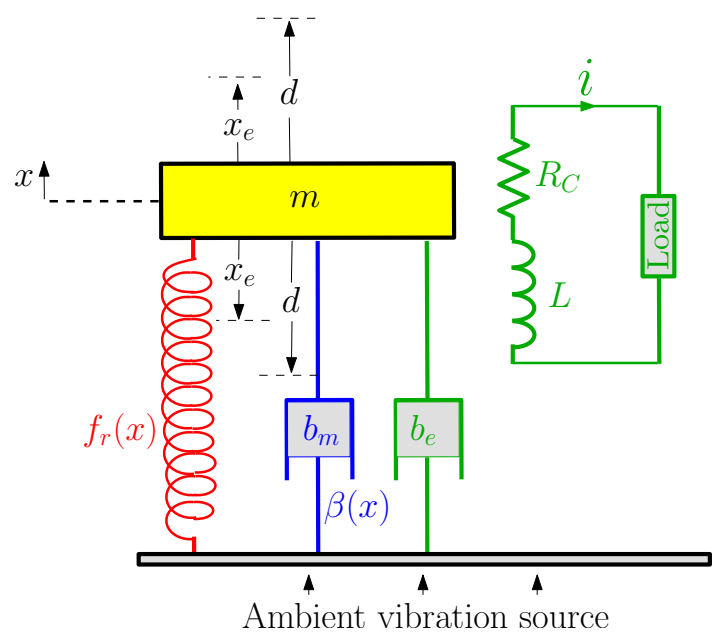

Fig. 1. Schematic diagram the spring-mass-damper system with spatial limitations. The system damping and stiffness change when the displacement reaches the value $\pm x_{e}$ and $\pm d$ respectively.

$\beta_{e}(x)$ depending on the state and a purely mechanical constant term $b_{m}$, i.e., $\beta(x)=\beta_{e}(x)+b_{m}$, where the term $\beta_{e}(x)$ is given by

$$
\beta_{e}(x)=\left\{\begin{array}{l}
b_{e} \text { if }|x| \leq x_{e} \\
0 \text { if }|x|>x_{e}
\end{array}\right.
$$

where $x_{e}$ is the length of the interval within which the electromechanical coupling is assumed to exist. The restoring force $f_{r}(x)$ is expressed as follows

$$
f_{r}(x)= \begin{cases}k(x+d) & \text { if } x<-d \\ 0 & \text { if }|x| \leq d \\ k(x-d) & \text { if } x>d\end{cases}
$$

where $d$ is the length of the interval within which the restoring force $f_{r}(x)$ acting on the inertial mass is assumed to be zero. Note that the stiffness corresponding to a linear spring can be restored from (3) by just putting $d=0$. Similarly, making $b_{e}=0$ in (2), one can recover the linear mechanical damping case.

\section{Dynamical model}

Let us consider that the system is driven with an external force $f(t)$. Therefore, the equation of the motion can be written as follows

$$
\ddot{x}+\frac{1}{m}\left(\beta(x) \dot{x}+f_{r}(x)\right)=\frac{f(t)}{m}
$$

where $x$ is the mass displacement, $\omega_{f}$ is the harmonic forcing angular frequency, and the overdot stands for taking the derivative with respect to time. Let $y=\dot{x}$ be the velocity of the mass. Then, (4) can be written in the following form:

$$
\begin{aligned}
& \dot{x}=y \\
& \dot{y}=-\frac{1}{m}\left(\beta(x) y+f_{r}(x)\right)+\frac{f(t)}{m}
\end{aligned}
$$

Different kinds of external vibrational source $f(t)$ can be considered. In some cases, these sources are governed by stochastic laws and their parameters can only be known in terms of statistical parameters such as mean values and variances [López-Suárez et al., 2011], [El Aroudi et al., 2013b]. However there are other applications where 
these sources can be considered deterministic signals such as in rotating machines and in vehicle and aircraft tires [Toh et al., 2007]. In the first case the idealized excitation sinusoidal term will only represent an approximation of the real case. For simplicity in the study of bifurcation phenomena and stability analysis a simple sinusoidal vibrational $T$-periodic source is considered, i.e, $f(t)=F \sin \left(\omega_{f} t\right)$, where $T=2 \pi / \omega_{f}$. Yet, when performing numerical simulations a Case study corresponding to a noisy vibrational source will be considered. The dynamics of the system is highly dependent on the system parameters. As the system is PWL, the dynamics for each system configuration can be described by a linear differential equation which can be written in the following form: $\dot{\mathbf{x}}=$ $\mathbf{A}_{i} \mathbf{x}+\mathbf{B}_{i}(t):=\mathbf{f}_{i}(\mathbf{x}, t)$, where $\mathbf{x}=(x, y)$ is the system vector of state variables and where $i=1 \ldots 8$ is an index whose value depends on the configuration that the system takes. It will be shown that in all cases $\mathbf{A}_{i}$ is a constant matrix while $\mathbf{B}_{i}(t)$ is a time varying periodic vector.

\subsection{Symmetry properties in the model}

Theorem 1. Let $\mathbf{x}_{1}(t)$ be solution of (5)-(6). Then, either $\mathbf{x}_{1}(t)$ is nonsymmetric and there exists another solution $\mathbf{x}_{2}(t)$ given by

$$
\mathbf{x}_{2}(t)=-\mathbf{x}_{1}\left(t \pm \frac{T}{2}\right)
$$

or $\mathbf{x}_{1}(t)$ is a unique symmetric solution with half period odd symmetry, i.e, $\mathbf{x}_{1}(t)=-\mathbf{x}_{1}\left(t \pm \frac{T}{2}\right)$.

Proof. Let us write the model (5)-(6) as follows:

$$
\dot{\mathbf{x}}=\mathbf{f}(\mathbf{x}, t)
$$

The proof is straightforward by using (5)-(6) and observing that $\mathbf{f}\left(-\mathbf{x}, t \pm \frac{T}{2}\right)=\mathbf{f}(\mathbf{x}, t)$

\subsection{System linear configurations}

Depending on the values of parameters $d$ and $x_{e}$, different sequences and configurations can take place. Figure 2 shows the possible cases depending on whether $d<x_{e}$ or $x_{e}<d, b_{e} \rightarrow 0$ or $d \rightarrow 0$. All possible transitions and their different conditions are represented in Fig. 3.

\subsubsection{Case 1: $x_{e}<d$}

This corresponds to Fig. 2(a). In this case, there are four different possible configurations $\left(\mathcal{C}_{1} \ldots \mathcal{C}_{4}\right)$ that the system can take. These configurations are

- Configuration $1\left(\mathcal{C}_{1}\right): f_{r}(x)=k(x+d)$ and $\beta(x)=b_{m}$. From (5)-(6), the system matrix $\mathbf{A}$ and vector $\mathbf{B}$ are

$$
\mathbf{A}_{1}=\left(\begin{array}{cc}
0 & 1 \\
-\frac{k}{m} & -\frac{b_{m}}{m}
\end{array}\right), \mathbf{B}_{1}=\left(\begin{array}{c}
0 \\
\frac{1}{m}\left(F \sin \left(\omega_{f} t\right)-k d\right)
\end{array}\right)
$$

- Configuration $2\left(\mathcal{C}_{2}\right): f_{r}(x)=0$ and $\beta(x)=b_{m}$. From (5)-(6), the system matrix $\mathbf{A}$ and vector $\mathbf{B}$ are

$$
\mathbf{A}_{2}=\left(\begin{array}{cc}
0 & 1 \\
0 & -\frac{b_{m}}{m}
\end{array}\right), \mathbf{B}_{2}=\left(\begin{array}{c}
0 \\
\frac{F}{m} \sin \left(\omega_{f} t\right)
\end{array}\right)
$$

- Configuration $3\left(\mathcal{C}_{3}\right): f_{r}(x)=0$ and $\beta(x)=b_{m}+b_{e}$. From (5)-(6), the system matrix $\mathbf{A}$ and vector $\mathbf{B}$ are

$$
\mathbf{A}_{3}=\left(\begin{array}{cc}
0 & 1 \\
0 & -\frac{b_{m}+b_{e}}{m}
\end{array}\right), \mathbf{B}_{3}=\left(\begin{array}{c}
0 \\
\frac{F}{m} \sin \left(\omega_{f} t\right)
\end{array}\right)
$$

- Configuration $4\left(\mathcal{C}_{4}\right): f_{r}(x)=k(x-d)$ and $\beta(x)=b_{m}$. From (5)-(6), the system matrix $\mathbf{A}$ and vector $\mathbf{B}$ are

$$
\mathbf{A}_{4}=\left(\begin{array}{cc}
0 & 1 \\
-\frac{k}{m} & -\frac{b_{m}}{m}
\end{array}\right), \mathbf{B}_{4}=\left(\begin{array}{c}
0 \\
\frac{1}{m}\left(F \sin \left(\omega_{f} t\right)+k d\right)
\end{array}\right)
$$




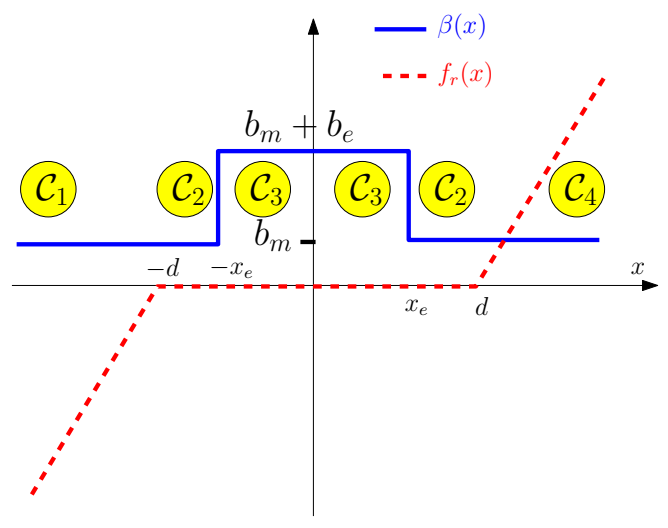

(a)

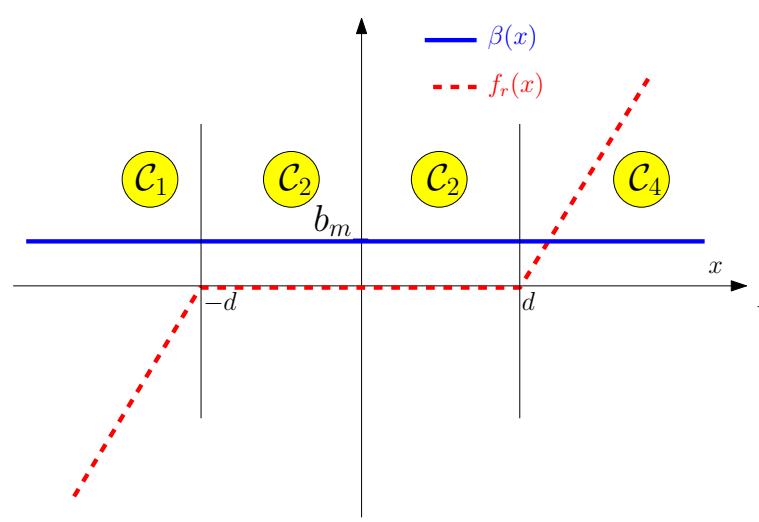

(c)

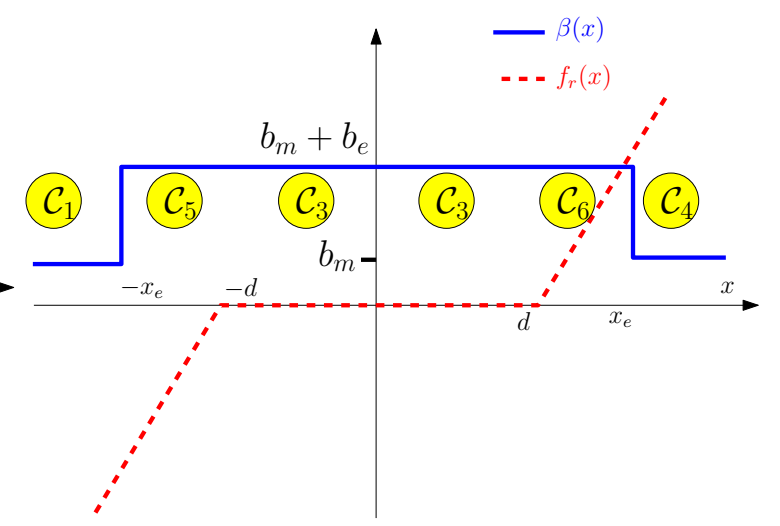

(b)

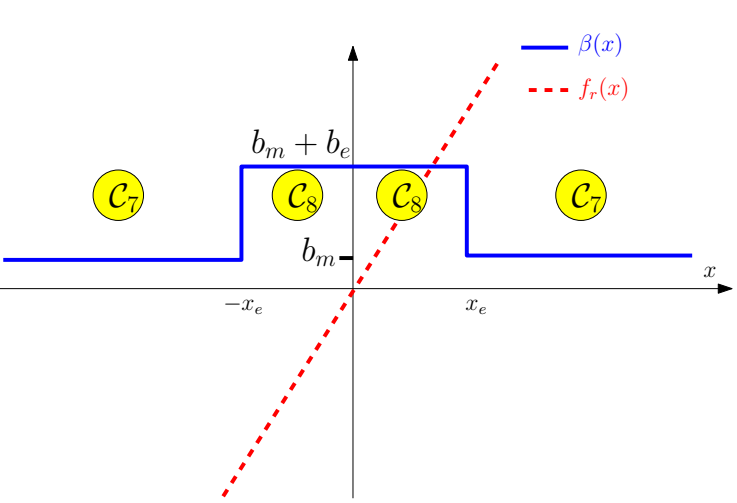

(d)

Fig. 2. Damping function $\beta(x)$ and restoring force $f_{r}(x)$. (a) $d>x_{e}$, (b) $d<x_{e}$, (c) $b_{e}=0$, (d) $d=0$.

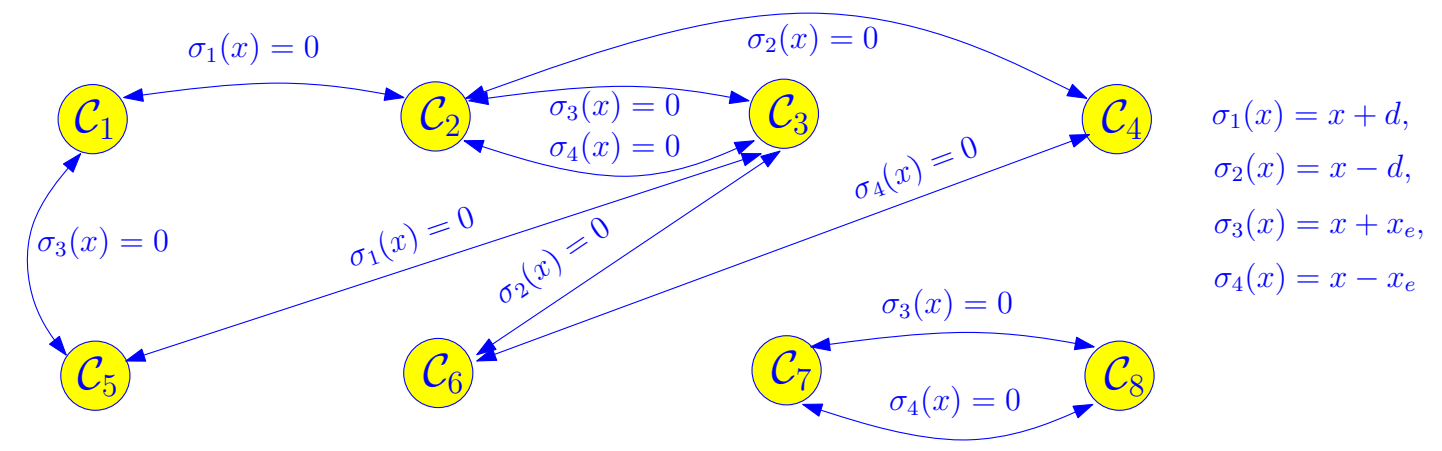

Fig. 3. Transition state diagram between the different subsystems and the underlying conditions.

\subsubsection{Case 2: $d<x_{e}$}

This corresponds to Fig. 2(b). In this case, in addition to the previous configurations, two new ones appear and Configuration $\mathcal{C}_{2}$ disappears. The configurations for this case are given as follows

- Configuration $5\left(\mathcal{C}_{5}\right): f_{r}(x)=k(x+d)$ and $\beta(x)=b_{m}+b_{e}$. From (5)-(6), the system matrix $\mathbf{A}$ and vector $\mathbf{B}$ are

$$
\mathbf{A}_{5}=\left(\begin{array}{cc}
0 & 1 \\
-\frac{k}{m} & -\frac{b_{m}+b_{e}}{m}
\end{array}\right), \mathbf{B}_{5}=\left(\begin{array}{c}
0 \\
\frac{1}{m}\left(F \sin \left(\omega_{f} t\right)-k d\right)
\end{array}\right)
$$

- Configuration $6\left(\mathcal{C}_{6}\right): f_{r}(x)=k(x-d)$ and $\beta(x)=b_{m}+b_{e}$. From (5)-(6), the system matrix $\mathbf{A}$ and vector $\mathbf{B}$ are 


$$
\mathbf{A}_{6}=\left(\begin{array}{cc}
0 & 1 \\
-\frac{k}{m} & -\frac{b_{m}+b_{e}}{m}
\end{array}\right), \mathbf{B}_{6}=\left(\begin{array}{c}
0 \\
\frac{1}{m}\left(F \sin \left(\omega_{f} t\right)+k d\right)
\end{array}\right)
$$

\subsubsection{Case 3: $b_{e} \rightarrow 0$}

This corresponds to Fig. 2(c). In this case, the system can only switch among three configurations which are Configurations $\mathcal{C}_{1}, \mathcal{C}_{2}$ and $\mathcal{C}_{4}$.

\subsubsection{Case 3: $d \rightarrow 0$}

This corresponds to Fig. 2(d). In this case, the system can switch between two new configurations which are Configurations $\mathcal{C}_{7}, \mathcal{C}_{8}$ given below

- Configuration $7\left(\mathcal{C}_{7}\right): f_{r}(x)=k x$ and $\beta(x)=b_{m}$. From (5)-(6), the system matrix $\mathbf{A}$ and vector $\mathbf{B}$ are

$$
\mathbf{A}_{7}=\left(\begin{array}{cc}
0 & 1 \\
-\frac{k}{m} & -\frac{b_{m}}{m}
\end{array}\right), \mathbf{B}_{7}=\left(\begin{array}{c}
0 \\
\frac{F}{m} \sin \left(\omega_{f} t\right)
\end{array}\right)
$$

- Configuration $8\left(\mathcal{C}_{8}\right): f_{r}(x)=k x$ and $\beta(x)=b_{m}+b_{e}$. From (5)-(6), the system matrix $\mathbf{A}$ and vector $\mathbf{B}$ are

$$
\mathbf{A}_{8}=\left(\begin{array}{cc}
0 & 1 \\
-\frac{k}{m} & -\frac{b_{m}+b_{e}}{m}
\end{array}\right), \quad \mathbf{B}_{8}=\left(\begin{array}{c}
0 \\
\frac{F}{m} \sin \left(\omega_{f} t\right)
\end{array}\right)
$$

As mentioned above, the linear case can be recovered from this PWL system by just making $b_{e} \rightarrow 0$ and $d \rightarrow 0$. In this case, the frequency response can be expressed in closed form by the following equations corresponding to the displacement and the velocity respectively

$$
\begin{gathered}
X(j \omega)=\frac{\frac{F}{m}}{-\omega^{2}+\frac{b}{m} j \omega+\frac{k}{m}} \\
Y(j \omega)=j \omega \frac{\frac{F}{m}}{-\omega^{2}+\frac{b}{m} j \omega+\frac{k}{m}}
\end{gathered}
$$

These expressions will be used to compare the frequency response of the PWL system with the linear equivalent system.

\subsection{Closed-form solutions corresponding to the different linear configurations}

Note that the system for each configuration can be described by a linear differential equation with a periodic input. Let us define the vectors $\mathbf{B}$ and $\mathbf{B}_{d}$ as follows

$$
\mathbf{B}=\left(\begin{array}{c}
0 \\
\frac{F}{m}
\end{array}\right), \quad \mathbf{B}_{d}=\left(\begin{array}{c}
0 \\
\pm \frac{k d}{m}
\end{array}\right)
$$

where the positive sign in the expression of $\mathbf{B}_{d}$ applies for Configurations $\mathcal{C}_{4}$ and $\mathcal{C}_{6}$ and the negative one applies for Configurations $\mathcal{C}_{1}$ and $\mathcal{C}_{5}$. For other configurations, the corresponding vector $\mathbf{B}_{d}$ is zero. The solution for the trajectory $\mathbf{x}(t)$ corresponding to Configuration $\mathcal{C}_{i}$ which takes place starting from an initial time instant $t_{i}$ with an initial state $\mathbf{x}\left(t_{i}\right)$ can be expressed as follows

$$
\mathbf{x}(t)=e^{\mathbf{A}_{i}\left(t-t_{i}\right)} \mathbf{x}\left(t_{i}\right)+\int_{t_{i}}^{t} e^{\mathbf{A}_{i}(t-\tau)} \mathbf{B}_{i}(\tau) \mathrm{d} \tau:=\boldsymbol{\Phi}_{i}\left(t-t_{i}\right) \mathbf{x}\left(t_{i}\right)+\mathbf{\Psi}_{i}\left(t, t_{i}\right)
$$


where the matrix $\boldsymbol{\Phi}_{i}$ is the state transition matrix corresponding to configuration $\mathcal{C}_{i}$ that can be expressed as follows

$$
\boldsymbol{\Phi}_{i}(t)=e^{\mathbf{A}_{i} t}
$$

while the vector $\Psi_{i}$ is given by

$$
\begin{aligned}
\mathbf{\Psi}_{i}\left(t, t_{i}\right) & =\left(\mathbf{A}_{i}^{2}+\omega_{f}^{2} \mathbf{I}\right)^{-1}\left(\boldsymbol{\Phi}_{i}\left(t-t_{i}\right)\left(\omega_{f} \cos \left(\omega_{f} t_{i}\right)+\mathbf{A}_{i} \sin \left(\omega_{f} t_{i}\right)\right)\right. \\
& \left.-\omega_{f} \mathbf{I} \cos \left(\omega_{f} t\right)-\mathbf{A}_{i} \sin \left(\omega_{f} t\right)\right) \mathbf{B}+\mathbf{A}^{-1}\left(\boldsymbol{\Phi}_{i}\left(t-t_{i}\right)-\mathbf{I}\right) \mathbf{B}_{d}
\end{aligned}
$$

\section{Stability analysis of periodic orbits in the PWL system}

Different approaches can be used to perform the stability analysis of the system under study. We will present here three different methods. The first approach will be based on a Poincaré map function [El Aroudi et al., 2007]. The second approach is the Floquet theory ([Aizerman and Gantmakher, 1958]) together with Fillipov methods for systems with discontinuous vector field [Fillipov, 1988], [Leine and Nijemeijer, 2004], [Giaouris et al., 2008]. The third approach is based on the FDM together with Floquet theory [Nayfeh et al., 2009], [Nayfeh and Balachandran, 1995].

\subsection{Poincaré map modeling}

Let us consider that within an interval of time $(n T,(n+1) T),(n \in \mathbb{N})$ a system trajectory is composed by $N_{c}$ different possible configurations of the system, where $T=2 \pi / \omega_{f}$ is the period of the external force. Let us also consider that the switching from one configuration to another occurs at time instants $t_{i, n}, i=1 \ldots N_{c}$, when a threshold condition in the form $\sigma_{i}\left(\mathbf{x}\left(t_{i, n}\right), t_{i, n}\right)=0$ is fulfilled. More specifically, let us suppose that the system take the following configurations during their corresponding time subintervals within an entire cycle $(n T,(n+1) T)$

$$
\begin{array}{ccr}
\text { Configuration } 1\left(\mathcal{C}_{1}\right): \dot{\mathbf{x}}=\mathbf{A}_{1} \mathbf{x}+\mathbf{B}_{1}(t):=\mathbf{f}_{1}(\mathbf{x}, t) & \text { for } & t \in\left[t_{0, n}, t_{1, n}\right] \\
\text { Configuration } 2\left(\mathcal{C}_{2}\right): \dot{\mathbf{x}}=\mathbf{A}_{2} \mathbf{x}+\mathbf{B}_{2}(t):=\mathbf{f}_{2}(\mathbf{x}, t) & \text { for } & t \in\left[t_{1, n}, t_{2, n}\right] \\
\vdots & & \\
\text { Configuration } i\left(\mathcal{C}_{i}\right): \dot{\mathbf{x}}=\mathbf{A}_{i} \mathbf{x}+\mathbf{B}_{i}(t):=\mathbf{f}_{i}(\mathbf{x}, t) & \text { for } \quad t \in\left[t_{i-1, n}, t_{i, n}\right]
\end{array}
$$

where $\mathbf{A}_{i}$ and $\mathbf{B}_{i}$ are the system matrices during phase $i\left(i=1,2, \ldots N_{c}\right)$. In all the cases, while $\mathbf{A}_{i}$ is a constant matrix, $\mathbf{B}_{i}$ is a time varying periodic vector. $\mathbf{x}$ is the vector of the state variables. The switching conditions can be written in the following compact form

$$
\sigma\left(\mathbf{x}_{n}, \mathbf{t}_{n}\right):=\left(\begin{array}{l}
\sigma_{1}\left(\mathbf{x}_{n}, t_{1}\right) \\
\sigma_{2}\left(\mathbf{x}_{n}, t_{1}, t_{2}\right) \\
\vdots
\end{array}\right)=\left(\begin{array}{l}
\mathbf{K} \mathbf{x}\left(t_{1, n}\right)-x_{1} \\
\mathbf{K} \mathbf{x}\left(t_{2, n}\right)-x_{2} \\
\vdots
\end{array}\right)=\left(\begin{array}{l}
0 \\
0 \\
\vdots
\end{array}\right)
$$

where $\mathbf{t}_{n}=\left[t_{1, n}, t_{2, n} \ldots, t_{i, n} \ldots,\right]^{\top}$ is the vector of switching instants within the $n t h$ cycle, $\mathbf{K}=\left[\begin{array}{ll}1 & 0\end{array}\right]$ is a suitable vector to select the displacement state variable $x$ from the complete state vector $\mathbf{x}$, (Note that $\mathbf{K} \mathbf{x}=x$ ) and $x_{i}$ is suitable boundary value for taking into account space limitations. Note that $x_{i}$ can take only the values of $\pm d$ or $\pm x_{e}$. By staking-up the solutions during their corresponding switching intervals, a Poincaré map can be built to carry out orbital stability analysis of different periodic orbits. The general expression of the map is as follows

$$
\begin{aligned}
\mathbf{x}_{n+1} & =\mathbf{f}\left(\mathbf{x}_{n}, \mathbf{t}_{n}\right) \\
\sigma\left(\mathbf{x}_{n}, \mathbf{t}_{n}\right) & =\mathbf{0}
\end{aligned}
$$

where $\mathbf{x}_{n}:=\mathbf{x}(n T)$ is the vector of the state variables at time instants $n T, n=1,2, \ldots$ The functions $\mathbf{f}$ and $\sigma$ can be determined by stacking-up the solutions during one switching cycle. 
To simplify notation, let $t_{n, i}=t_{i}$. The mapping $\mathbf{f}$ in (25) that relates the state variable $\mathbf{x}_{n}$ at the beginning of an entire cycle to $\mathbf{x}_{n+1}$, that at the end of the same cycle, can expressed as follows

$$
\mathbf{f}\left(\mathbf{x}_{n}, \mathbf{t}_{n}\right)=\prod_{i=M}^{1} \mathbf{\Phi}_{i}\left(t_{i}-t_{i-1}\right) \mathbf{x}_{n}+\sum_{j=1}^{M-1} \prod_{i=M}^{j+1} \mathbf{\Phi}_{i}\left(t_{i}, t_{i-1}\right) \mathbf{\Psi}_{j}\left(t_{j}, t_{j-1}\right)+\mathbf{\Psi}_{N_{c}}\left(T, t_{N_{c}-1}\right)
$$

while by using (20) the general switching constraint $\sigma\left(\mathbf{x}_{n}, \mathbf{t}_{n}\right)=0$ in (26) can be written in the following form

$$
\sigma\left(\mathbf{x}_{n}, \mathbf{t}_{n}\right)=\left(\begin{array}{l}
\mathbf{K}\left(\boldsymbol{\Phi}_{1}\left(t_{1}\right) \mathbf{x}_{n}+\mathbf{\Psi}_{i}\left(t_{1}\right)\right)-x_{1} \\
\mathbf{K}\left(\boldsymbol{\Phi}_{2}\left(t_{2}-t_{1}\right)\left[\boldsymbol{\Phi}_{1}\left(t_{1}\right) \mathbf{x}_{n}+\Psi_{1}\left(t_{1}\right)\right]+\mathbf{\Psi}_{2}\left(t_{2}, t_{1}\right)\right)-x_{2} \\
\vdots
\end{array}\right)=\left(\begin{array}{l}
0 \\
0 \\
\vdots
\end{array}\right)
$$

The map (27) and the constraint (28) define a generalized Poincaré map for a PWL switching system with $N_{c}$ configurations.

\subsubsection{Fixed points}

The fixed points $\mathbf{X}$ of the map correspond to periodic orbits of the PWL system. They can be obtained by enforcing the periodicity: $\mathbf{x}_{n}=\mathbf{x}_{n+1}=\mathbf{X}$. Using the expression of $\mathbf{P}, \mathbf{X}$ can be expressed in terms of switching time instants $t_{i}$ and system matrices $\boldsymbol{\Phi}_{i}$ and vectors $\boldsymbol{\Psi}_{i}$. Enforcing periodicity in (27) and solving for $\mathbf{X}$, one obtains that the fixed point can be expressed as follows

$$
\mathbf{X}=\left(\mathbf{I}-\prod_{i=N_{c}}^{1} \mathbf{\Phi}_{i}\left(t_{i}, t_{i-1}\right)\right)^{-1}\left(\sum_{j=1}^{N_{c}-1} \prod_{i=N_{c}}^{j+1} \boldsymbol{\Phi}_{i}\left(t_{i}, t_{i-1}\right) \mathbf{\Psi}_{j}\left(t_{j}, t_{j-1}\right)+\mathbf{\Psi}_{N_{c}}\left(T, t_{N_{c}-1}\right)\right)
$$

where $\mathbf{I}$ is an identity matrix with appropriate size. In the steady state $\mathbf{t}_{n}=\mathbf{t}$ is the vector of switching instants corresponding to the fixed point $\mathbf{X}$. To obtain these instants, we can replace $\mathbf{x}_{n}$ in (28) by $\mathbf{X}$ using the expression in (29) and we obtain the general switching condition $(\sigma(\mathbf{t})=0)$ depending only on $\mathbf{t}$. Solving this vectorial equation for $\mathbf{t}$ (recall that $t_{i}=n T$ and $t_{n+1, i}=(n+1) T$ are known), we can replace $\mathbf{t}$ in (29) and obtain the fixed points of the map. In general, the solution of the equation $(\sigma(\mathbf{t})=0)$ is not available in closed-form since this equation is transcendental. Therefore a root-finding algorithm should be applied. Once the fixed points are located, their stability analysis may be carried out by obtaining the Jacobian matrix $\mathbf{J}$ and studying the local behavior of the map $\mathbf{P}$ near these fixed points. In the next subsection the Jacobian matrix corresponding to the PWL system is obtained.

\subsubsection{Jacobian matrix and stability analysis of nominal periodic orbits}

The stability of fixed points $\mathbf{X}$, of the map $\mathbf{f}$, can be investigated using the Jacobian matrix $\mathbf{J}$. This matrix is obtained in closed form in terms of the switching instants $t_{i}$. The expression of the Jacobian matrix $\mathbf{J}$ can be expressed as follows

$$
\mathbf{J}=\frac{\partial \mathbf{f}}{\partial \mathbf{x}_{n}}+\frac{\partial \mathbf{f}}{\partial \mathbf{t}_{n}} \frac{\mathbf{d} \mathbf{t}_{n}}{\mathbf{d} \mathbf{x}_{n}}
$$

where we have assumed that all parameters are constants during the entire cycle. Differentiating (26), we can write

$$
\mathbf{0}=\frac{\partial \sigma}{\partial \mathbf{x}_{n}} \mathbf{d} \mathbf{x}_{n}+\frac{\partial \sigma}{\partial \mathbf{t}_{n}} \mathbf{d t}_{n}
$$

from which we can deduce $\frac{\mathbf{d t} \mathbf{t}_{n}}{\mathbf{d x _ { n }}}$ and replace its expression in (30) to obtain

$$
\mathbf{J}=\frac{\partial \mathbf{f}}{\partial \mathbf{x}_{n}}-\frac{\partial \mathbf{f}}{\partial \mathbf{t}_{n}}\left(\frac{\mathbf{d} \sigma}{\mathbf{d t}_{n}}\right)^{-1} \frac{\mathbf{d} \sigma}{\mathbf{d} \mathbf{x}_{n}}
$$

By calculating each term in (31), the Jacobian matrix $\mathbf{J}$ can be obtained in a straightforward manner (see [El Aroudi et al., 2007] for more details). Evaluating this Jacobian matrix in a fixed point and computing its corresponding eigenvalues $\lambda_{i}$ would give us the stability of its underlying periodic orbit. If $\mathbf{J}$ has all eigenvalues within the unit circle, the periodic orbit is stable (no bifurcating or hyperbolic orbit). If an eigenvalue crosses the unit circle from inside to outside, the periodic orbit loses its stability and this is a sign of a bifurcation. 


\subsection{Floquet theory and Fillipov method}

Another approach for stability analysis of PWL systems is based on the Floquet theory and the eigenvalues of the fundamental solution matrix over one entire cycle. This matrix is also called the monodromy matrix. For piecewise smooth systems, as is the case for the system considered in this study, the monodromy matrix can be constructed from the product of the state transition matrices corresponding to each sub-cycle and the corresponding saltation matrix [Leine and Nijemeijer, 2004].

Let $\mathbf{x}$ be the vector of state variables as defined previously, and during a switching cycle, let $t_{i}$ be the switching instant corresponding to when the system changes its dynamics from Configuration $\mathcal{C}_{i}$ to $\mathcal{C}_{i+1}$. For each configuration, the system equations are linear and time invariant. An approach for stability analysis of piecewise linear systems is based on the Floquet theory and the eigenvalues of the monodromy matrix. The eigenvalues of this matrix are called Floquet multipliers. For piecewise linear systems, as is the case for the system considered in this study, the monodromy matrix can be constructed from the product of the state transition matrices corresponding to each sub-cycle and the corresponding saltation matrix [Leine and Nijemeijer, 2004; Aizerman and Gantmakher, 1958; Fillipov, 1988; Giaouris et al., 2008]. Suppose a trajectory $\mathbf{x}(t)$ starts at time instant $t_{i}$ and is passing from the Configuration $\mathcal{C}_{i}$ described by the vector field $\mathbf{A}_{i} \mathbf{x}+\mathbf{B}_{i}(t):=\mathbf{f}_{i}(\mathbf{x}, t)$, intersects the switching boundary described by the equation $\sigma_{i}(\mathbf{x}, t)=0$ at $t_{i}$, and goes to Configuration $\mathcal{C}_{i+1}$ given by the vector field $\mathbf{A}_{i+1} \mathbf{x}+\mathbf{B}_{i+1}(t):=\mathbf{f}_{i+1}(\mathbf{x}, t)$. It has been shown, using the Fillipov method ([Fillipov, 1988; Leine and Nijemeijer, 2004; Giaouris et al., 2008]), that when there is a transversal intersection, the state transition matrix across the switching boundary, called also the saltation matrix $\mathbf{S}_{i}$, is given by

$$
\mathbf{S}_{i}=\mathbf{I}+\frac{\left(\mathbf{f}_{i+1}\left(\mathbf{x}\left(t_{i}\right)\right)-\mathbf{f}_{i}\left(\mathbf{x}\left(t_{i}\right)\right)\right) \mathbf{K}_{i}}{\mathbf{K}_{i} \mathbf{f}_{i}(\mathbf{x})+\left.\frac{\partial \sigma_{i}}{\partial t}\right|_{t=t_{i}}}
$$

where $\mathbf{I}$ is a unitary matrix with appropriate size. Then, the monodromy matrix $\mathbf{M}$ for a piecewise linear system with $N_{c}$ different configurations can be composed during a complete cycle as follows

$$
\mathbf{M}(\mathbf{x}(0))=\boldsymbol{\Phi}_{N_{c}} \mathbf{S}_{N_{c}-1} \ldots \boldsymbol{\Phi}_{2} \mathbf{S}_{1} \boldsymbol{\Phi}_{1}
$$

where $\boldsymbol{\Phi}_{i}=\boldsymbol{\Phi}_{i}\left(t_{i}-t_{i-1}\right)=e^{\mathbf{A}_{i}\left(t_{i}-t_{i-1}\right)}, i=1 \ldots N_{c}$, is the state transition matrix corresponding to the interval $\left(t_{i-1}, t_{i}\right)$ within which Configuration $\mathcal{C}_{i}$ is taking place. It is worth noting that the previous approach can be applied to periodic orbits with any period. In this paper we have used this approach for studying the stability of $T$-periodic and $2 T$-periodic orbits for different operating sequences characterized both by different values of $N_{c}$. It is also worth noting that the monodromy matrix $\mathbf{M}(\mathbf{x}(0))$ turns out to coincide with the Jacobian matrix $\mathbf{J}$ of the Poincaré map evaluated at the fixed point $\mathbf{x}(0)$ which in turn coincides with the fixed point $\mathbf{X}$ given in (29) in the case of a stroboscopic Poincaré mapping.

\subsection{Finite Difference Method (FDM)}

The analytical methods which has been presented above depends essentially on the assumption that the system switches according to a well known and fixed sequence, an assumption which is justifiable if under parameter changes, the same sequence holds. One would be like to be able to know under which set of parameter values the system sequence can be altered. The difficulty is that one cannot hope to have a general result embracing all the cases. It might be possible, however, to deal with stability analysis without assuming any switching sequence using the FDM.

In the FDM [Nayfeh et al., 2009], in order to examine the periodic response of the PWL system to the harmonic loading, we discretize the orbit, whose period is identically equal to $T$, using $m+1$ equally-spaced points between time instants $t_{0}$ and $t_{m}$ and enforce the periodicity condition $\mathbf{x}_{0}=\mathbf{x}_{m}$, where $\mathbf{x}_{0}=\mathbf{x}\left(t_{0}\right)$ and $\mathbf{x}_{m}=\mathbf{x}\left(t_{m}\right)$. Such condition implies that the first and last points of the orbit (points $\mathbf{x}_{0}$ and $\mathbf{x}_{m}$ ) are identical. Consequently, the orbit is time-discretized using $m$ intervals. At each of these points, we have the following set of equations for $p=0 \ldots m$.

$$
\begin{aligned}
& \dot{x}_{p}=y_{p} \\
& \dot{y}_{p}=-\frac{1}{m}\left(\beta\left(x_{p}\right) y_{p}+f_{r}\left(x_{p}\right)\right)+\frac{F}{m} \sin \left(\omega_{f} t_{p}\right)
\end{aligned}
$$


The FDM can now be applied to system (34)-(35) to yield a set of nonlinear algebraic equations. In this case, a two-step explicit central-difference scheme is used to approximate the time derivatives [Nayfeh and Balachandran, 1995]. Therefore, for an $m+1$ FDM-discretized orbit, the PWL dynamics can be approximated by a set of $2 m$ nonlinear algebraic equations in $2 m$ unknown displacements and velocities. These equations can be solved for the unknowns using the Newton-Raphson method. The stability of each obtained orbit can then be found by combining FDM discretization with Floquet theory [Nayfeh et al., 2009].

\section{Numerical Results}

\subsection{Dimensionless parameters and state variables}

To reduce the number of bifurcation parameters let us define the following dimensionless parameters

$$
b_{m}^{\prime}=\frac{b_{m}}{m \omega_{0}}, \quad b_{e}^{\prime}=\frac{b_{e}}{m \omega_{0}}, \quad x_{e}^{\prime}=\frac{x_{e}}{d}, \quad F^{\prime}=\frac{F}{k d}, \quad \omega_{f}^{\prime}=\frac{\omega_{f}}{\omega_{0}}
$$

where $\omega_{0}=\sqrt{k / m}$. This dimensionless formulation allows us to decrease the number of parameters from eight to five. Let us also define the following dimensionless state variables and time

$$
t^{\prime}=\omega_{0} t, \quad x^{\prime}=\frac{x}{d}, \quad y^{\prime}=\frac{y}{d \omega_{0}}
$$

For convenience, in the following we drop the use of primes in the definition of dimensionless state variables and parameters.

\subsection{Bifurcation behavior and stability analysis of periodic solutions}

The value of the dimensionless excitation frequency considered in this section is $\omega_{f}=0.3$. Without loss of generality let us take the force intensity as a primary bifurcation parameter while the parameter $x_{e}$ will be considered as a secondary bifurcation parameter. Time-domain series trajectories in the state-space and bifurcation diagrams will be computed and bifurcation phenomena will be explained by using the Floquet multipliers. The frequency response of the system will also be computed and its performance represented by the average harvested power will be presented.

\subsubsection{Case study 1: $x_{e}=0.3, b_{m}=0.2$ and $b_{e}=0.4$}

Figure 4(a) shows the bifurcation diagram of the system by taking the amplitude $F$ of the external force as a bifurcation parameter. For $F<0.4$, the system exhibits a stable $T$-periodic orbit with a half period odd symmetry. At $F \approx 0.4$ a symmetry breaking or a pitchfork bifurcation takes place. At this critical value of the bifurcation parameter, the symmetric $T$-periodic orbit becomes unstable and two new non-symmetric stable $T$-periodic solutions emerge. These orbits can also undergo a period doubling bifurcation by further increasing $F$ leading to the appearance of two $2 T$-periodic orbits. In this case study, the first period doubling occurs at $F \approx 0.48$. Successive period doubling may lead to chaotic behavior. This occur for $F \approx 1$. The corresponding Floquet multipliers and the period of the periodic orbits are plotted in Fig. 4(b) where both kinds of instabilities can be identified by observing the values of the characteristic multipliers at the critical points. Namely, at a symmetry breaking bifurcation, one of the Floquet multipliers is equal to 1 while at a period doubling bifurcation one of these multipliers is equal to -1 . It can be observed that bubbles of bifurcations in the vicinity of $F \approx 1.3$ are sandwiched by period doubling bifurcations born from symmetry breaking or pitchfork bifurcation.

It is worth noting here that the three previously presented methods for computing the Floquet multipliers gave the same results. In the FDM, $m=100$ intervals are used for each forcing period and then equations (34)-(35) are integrated and Floquet multipliers are calculated according the approach explained in Section 4.3. Hereinafter, the systematic approach based on obtaining a Poincaré map and its Jacobian matrix is used after identifying the switching sequence of the orbit.

\subsubsection{Case study 2: $x_{e}=0.5, b_{m}=0.2$ and $b_{e}=0.4$}

All other parameters are the same as before except $x_{e}$ which is increased. The resulting bifurcation diagram is shown in Fig. 5. Comparing this case with the previous one, it can be observed that as the secondary bifurcation parameter 

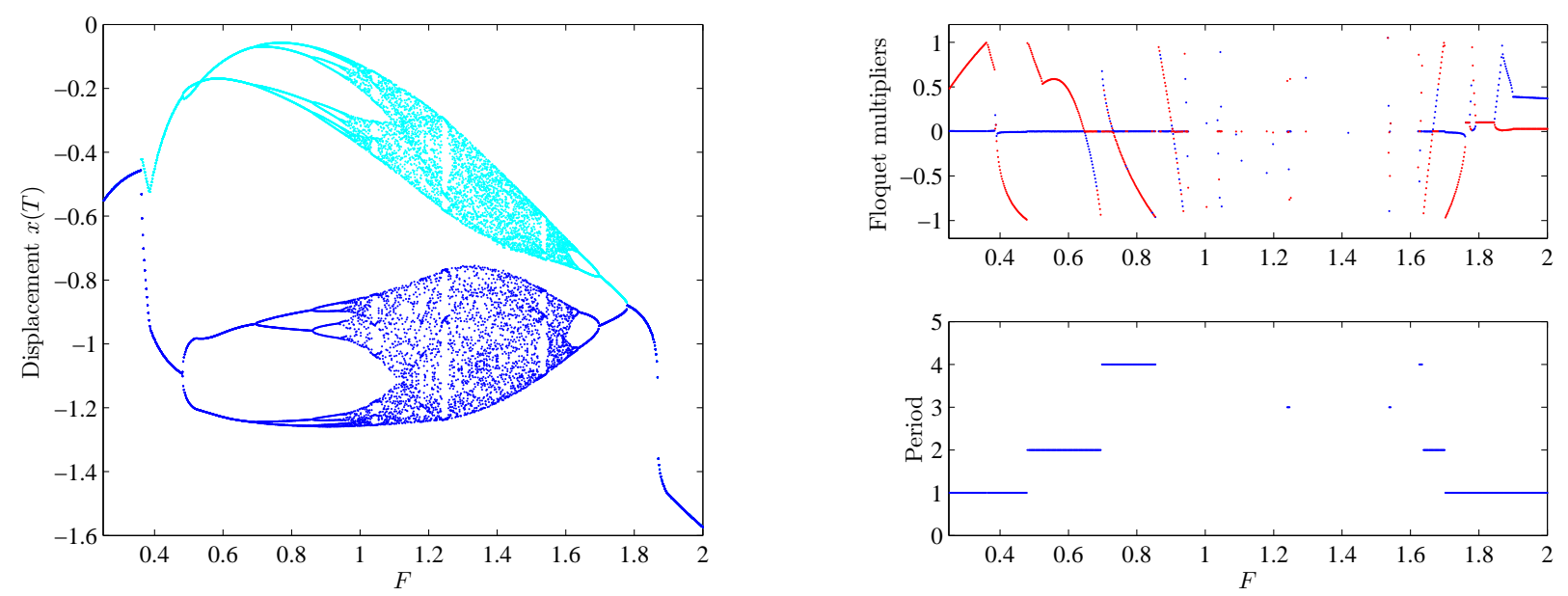

Fig. 4. Bifurcation diagrams, Floquet multipliers and fundamental period of the different stable periodic solutions of the PWL system taking $F$ as a bifurcation parameter for $x_{e}=0.3, b_{m}=0.2$ and $b_{e}=0.4$.
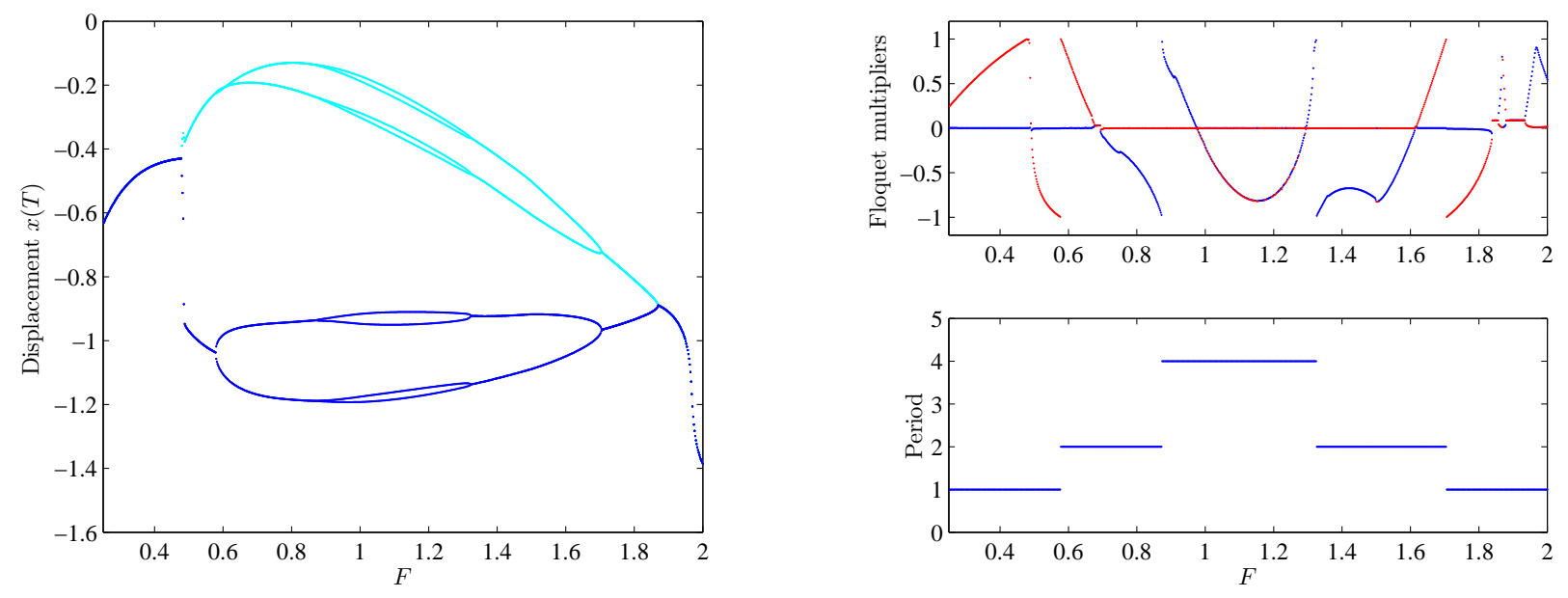

Fig. 5. Bifurcation diagrams, characteristic multipliers and fundamental period of the different stable periodic solutions of the PWL system taking $F$ as a bifurcation parameter for $x_{e}=0.5, b_{m}=0.2$ and $b_{e}=0.4$.

value is increased to $x_{e}=0.5$ some period doubling bifurcations and the associated chaotic regimes disappear and the system exhibits periodic orbits with different periods. Figure 6 and 7 show the time-domain waveforms and the state-space trajectories for different values of the bifurcation parameter $F$. When two stable orbits coexist, both are plotted in the same figure. Different switching sequences and motion regimes can be identified for the system:

- For $F=0.45$ the switching sequence is as follows

$$
\mathcal{C}_{3} \rightarrow \mathcal{C}_{2} \rightarrow \mathcal{C}_{4} \rightarrow \mathcal{C}_{2} \rightarrow \mathcal{C}_{3} \rightarrow \mathcal{C}_{2} \rightarrow \mathcal{C}_{1} \rightarrow \mathcal{C}_{2} \ldots
$$

For this value of $F$, the system periodic orbit is stable and exhibits a half period odd symmetry as it can be observed in the time-domain waveforms and in the system state-space trajectory (Fig. 6(a) and Fig. 7-(a)).

- As the parameter $F$ is increased, the unique stable periodic orbit exhibits a symmetry breaking or a pitchfork bifurcation at $F \approx 0.45$. Two new solutions emerge while the previous one loses its stability through a pitchfork bifurcation. Fig. 6(b) and Fig. 7(b) show the behavior of the system just after this bifurcation takes place. It can be observed that two different orbits coexist for the same set of parameter values. Moreover, one of the orbits is half period odd symmetric with respect to the other. This situation is more enhanced by increasing $F$ further (Fig. 6(c) 


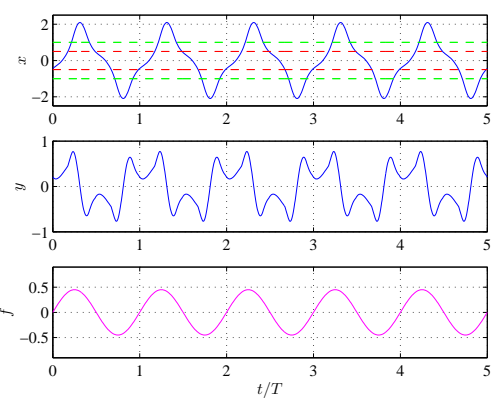

(a) $F=0.45$
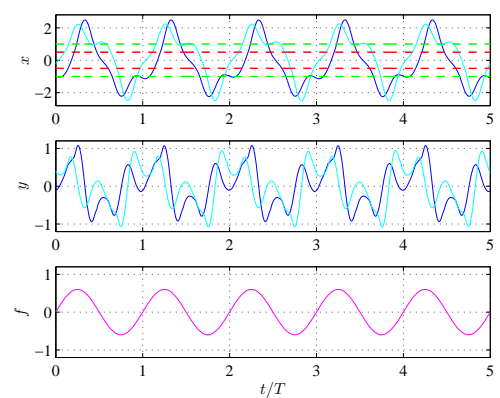

(d) $F=0.6$
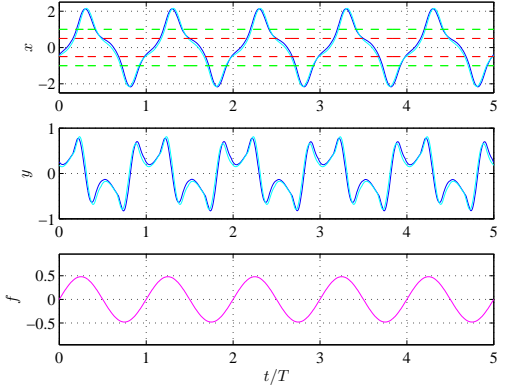

(b) $F=0.48$
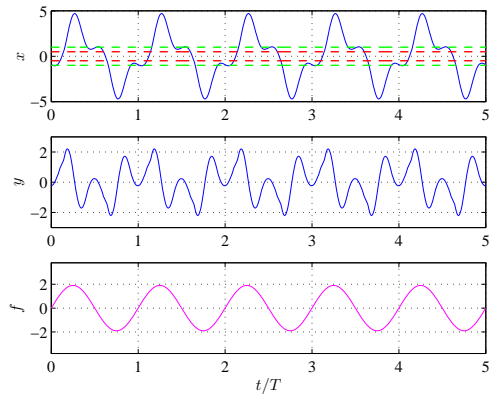

(e) $F=1.9$
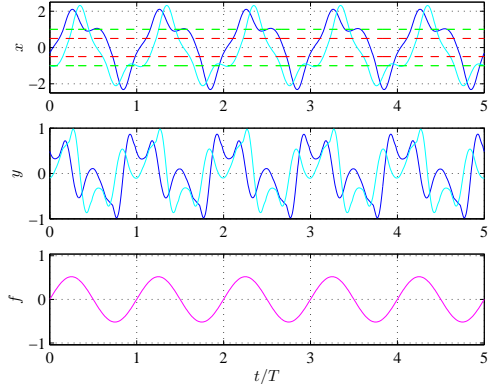

(c) $F=0.52$
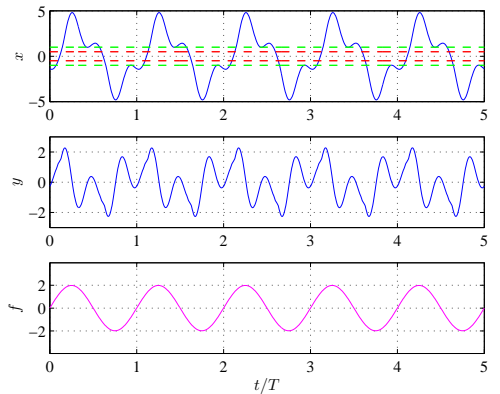

(f) $F=1.99$

Fig. 6. Time-domain waveforms for different values of $F$.

and Fig. 7(c)). The switching sequence for the two new emerged solutions are as follows

$$
\mathcal{C}_{3} \rightarrow \mathcal{C}_{2} \rightarrow \mathcal{C}_{4} \rightarrow \mathcal{C}_{2} \rightarrow \mathcal{C}_{4} \rightarrow \mathcal{C}_{2} \rightarrow \mathcal{C}_{3} \rightarrow \mathcal{C}_{2} \rightarrow \mathcal{C}_{1} \rightarrow \mathcal{C}_{2} \ldots
$$

- By increasing $F$ further both orbits undergo a period doubling bifurcation and two different $2 T$-periodic orbits emerge (Fig. 6(d) and Fig. 7(d)). This can be confirmed by the fact that one of the Floquet multipliers crosses the unit circle form the point $(-1,0)$ in the complex plane.

- This orbit can also undergo a period doubling bifurcation and a $4 T$-periodic orbit is exhibited.

Similar phenomena occur reversely as the the bifurcation parameter is increased till the $T$-periodic orbit becomes symmetric stable and unique as is illustrated in the time-domain waveforms of Fig. 6(e) and Fig. 6(f) and their corresponding state-space trajectories of Fig. 7(e) and Fig. 7(f).

\subsubsection{Case study 3: $x_{e}=1.2, b_{m}=0.2$ and $b_{e}=0.4$}

All other parameters are the same as before except $x_{e}$ which is further increased in this case to 1.2. The bifurcation diagram and the corresponding Floquet multipliers are plotted in Fig. 8. It can be observed that all period doubling bifurcations disappeared and only symmetry breaking bifurcation can take place.

\subsubsection{Case study 4: $x_{e}=10, b_{m}=0$ and $b_{e}=0.25$}

In this case, $x_{e}=10>>1$ in such a way that the corresponding boundary is not visited by the state variable $x$ and the number of configurations in a switching sequence is reduced. The parameters $b_{m}$ and $b_{e}$ are selected such that the total damping is constant and the only existing boundaries are the ones corresponding to the stiffness change taking place at $x= \pm 1$ in the dimensionless formulation. As before, the bifurcation diagram together with Floquet multipliers are plotted. These are shown in Figure 9. The results obtained in this figure is similar to Figure 4 since both of them correspond to practically the same average total damping. In fact, the damping in Figure 4 is a little bit larger than in Figure 9, and hence some instabilities disappear because of this extra damping. As in the case of Figure 4, pitchfork bifurcation takes place first resulting in symmetry breaking of the orbits. These undergo period 


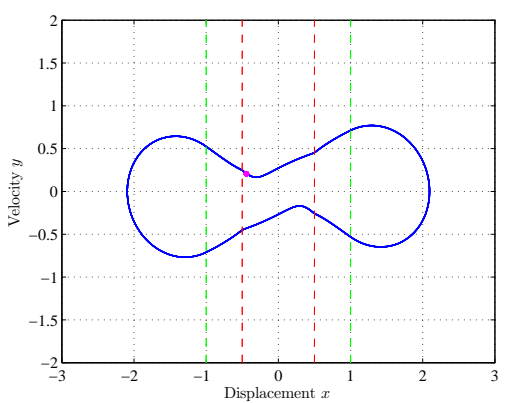

(a) $F=0.45$

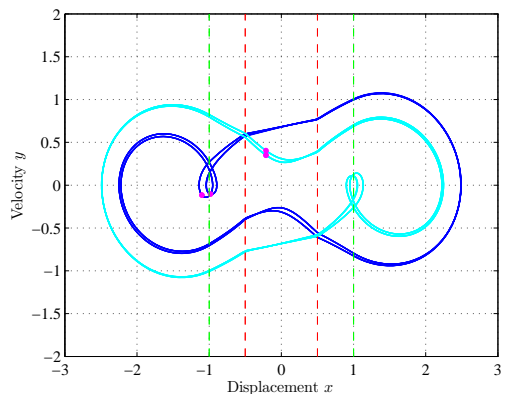

(d) $F=0.6$

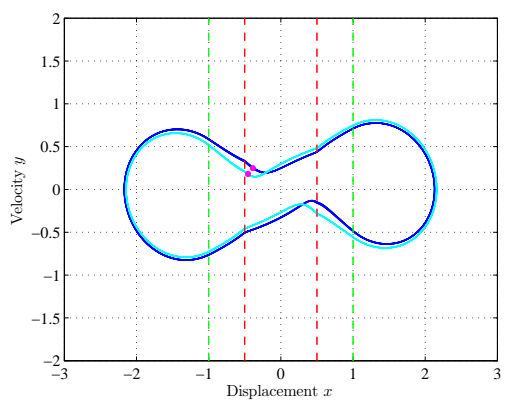

(b) $F=0.48$

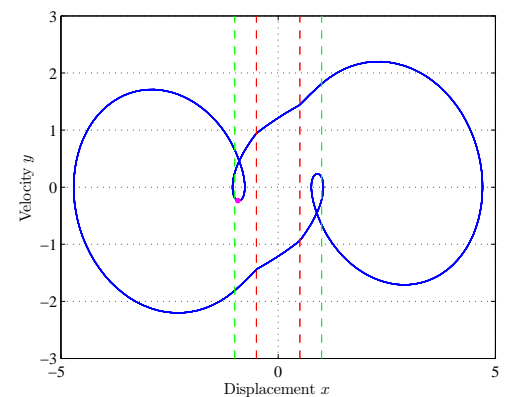

(e) $F=1.9$

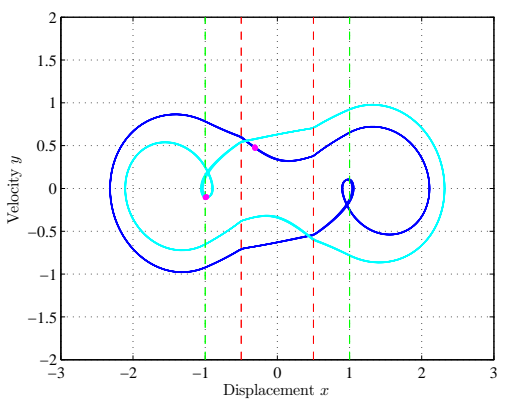

(c) $F=0.52$

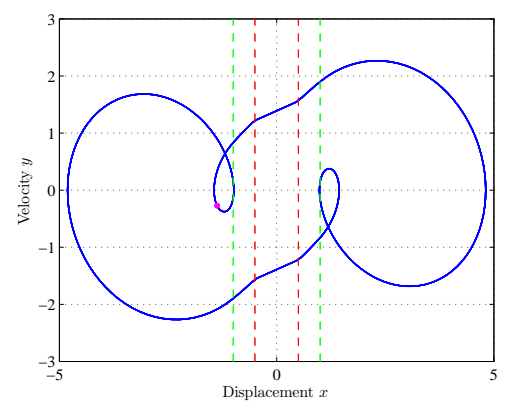

(f) $F=1.99$

Fig. 7. Trajectories of the PWL system for different values of $F$. Boundaries are also shown in dashed lines.
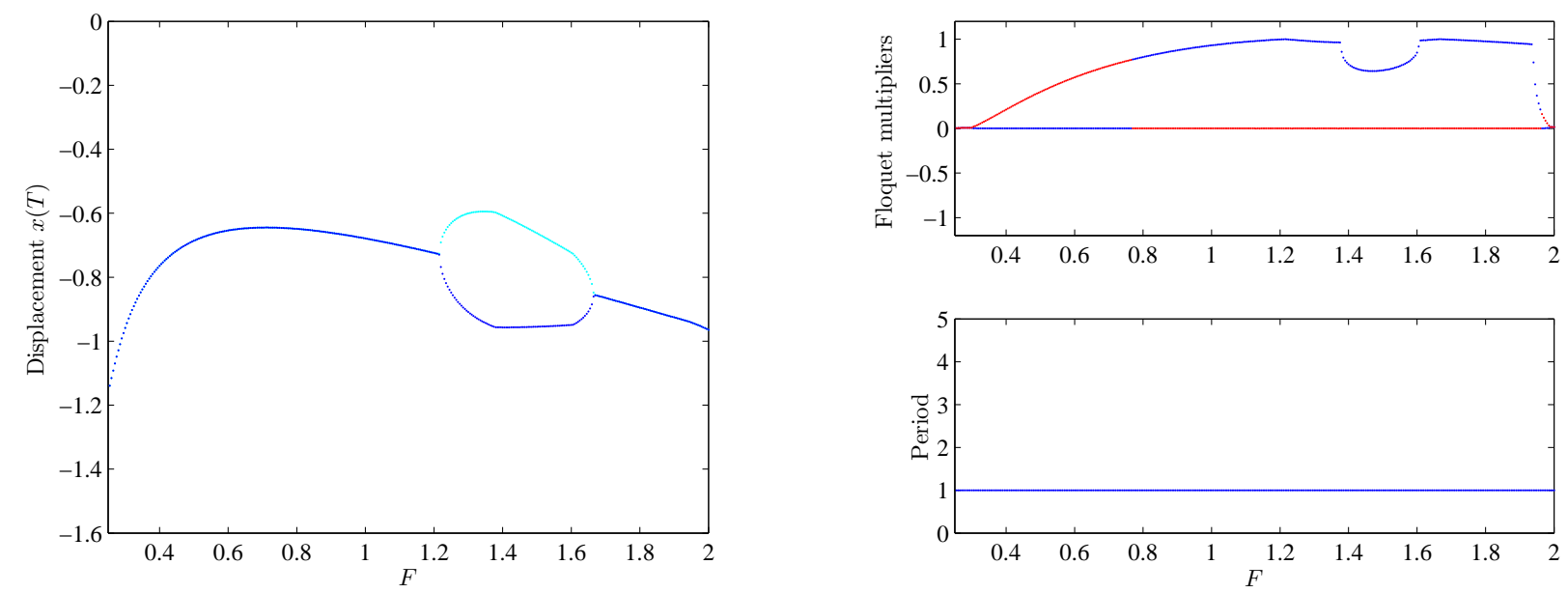

Fig. 8. Bifurcation diagram, Floquet multipliers and fundamental period of the stable periodic solutions of the PWL system taking $F$ as a bifurcation parameter for $x_{e}=1.2, b_{m}=0.2$ and $b_{e}=0.4$.

doubling bifurcation when the bifurcation parameter is increased. Bubbles of bifurcation sandwiched by period doubling bifurcations born from symmetry breaking bifurcation can also be observed. These results show that the influence of $x_{e}$ is merely quantitative and its effect is equivalent to the one observed if the damping is modified.

\subsection{Performances of the system in terms of the harvested power}

In this section the performance of the system as an energy harvester will be studied and will be compared with a linear counterpart. First a purely harmonic excitation is considered. Then a noisy vibrational input will be used as an external excitation for the PWL system. 

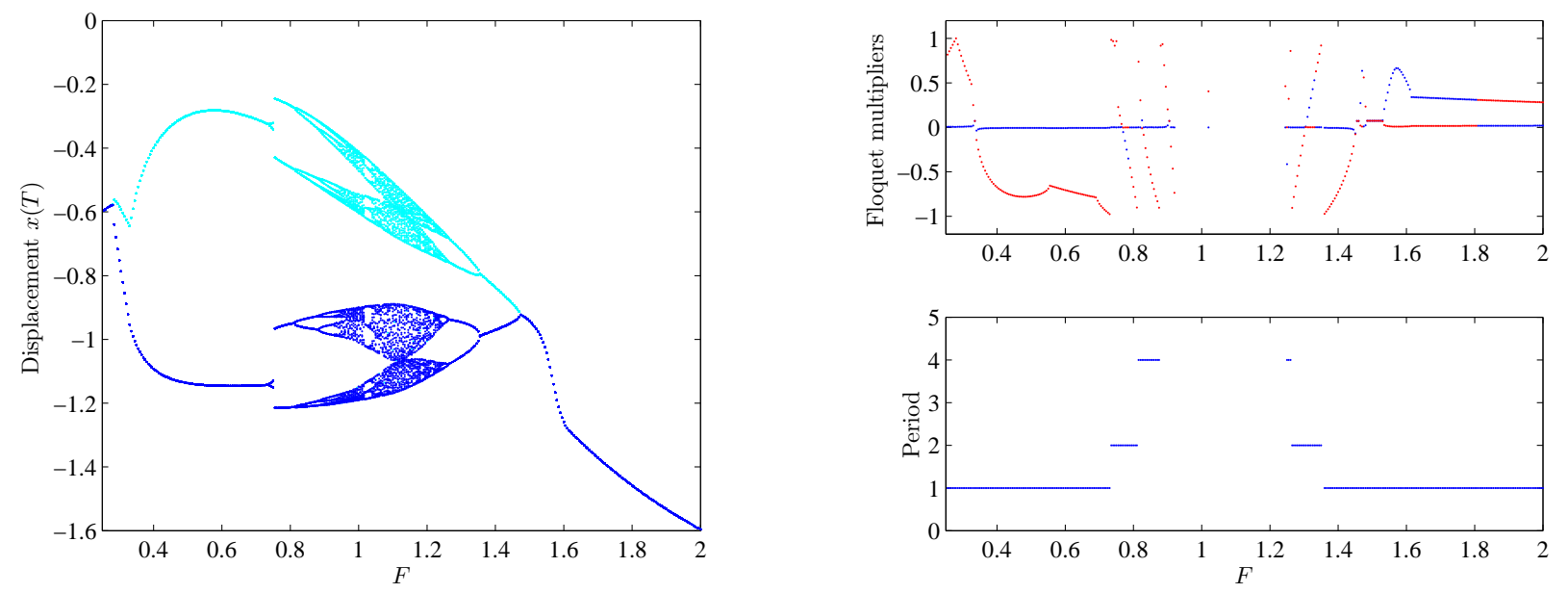

Fig. 9. Bifurcation diagram, Floquet multipliers and fundamental period of the different stable periodic solutions of the PWL system taking $F$ as a bifurcation parameter for $x_{e}=10, b_{m}=0$ and $b_{e}=0.25$.

\subsubsection{Purely harmonic excitation}

In this case, the excitation angular frequency is swept in the low frequency range $(0.1,1)$ while the base excitation intensity is varied in the range $(0.25,1)$. Without loss of generality, case study 2 is considered corresponding to $x_{e}=0.5, b_{m}=0.2$ and $b_{e}=0.4$.

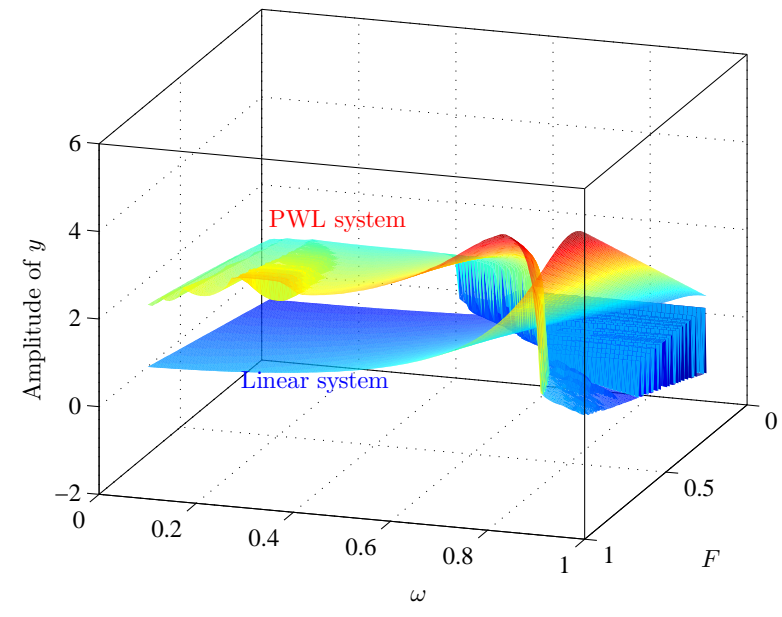

(a)

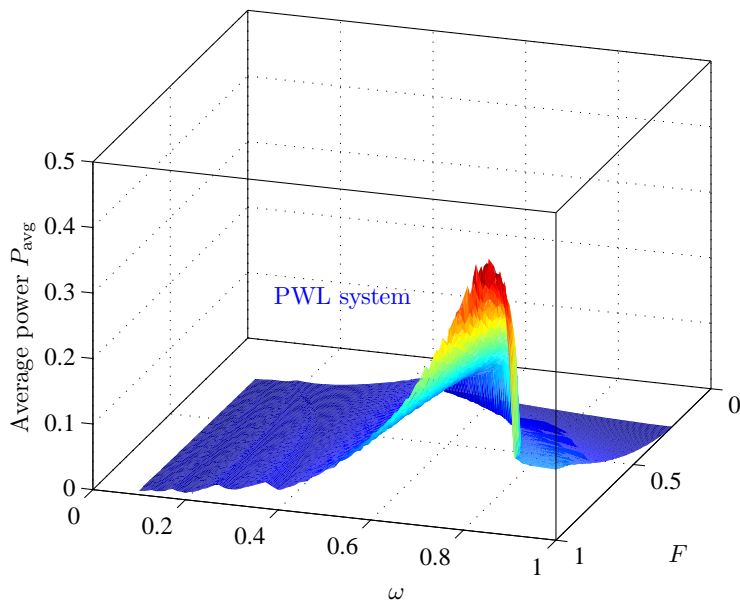

(b)

Fig. 10. Numerically obtained velocity amplitude responses of the PWL system and the analytical frequency response of the linear equivalent system (a) and the average harvested power of the PWL system (b) for different values of the force intensity $F$ and frequency $\omega_{f}$.

A mesh plot of the amplitude of the velocity is depicted in Fig. 10 for the PWL system. For comparison, the frequency response of an equivalent linear system ([Stephen, 2011]) is also plotted in the same figure. The system with PWL stiffness and damping factor has higher amplitude of velocities for low values of external forcing frequencies $\omega_{f}$ specially for low values of force intensity $F$ (Fig. 11(a)). This could be an advantage for potential use of this system in low frequency vibrational-based energy as it is the case with nonlinear energy harvesters with smooth nonlinearities inducing double well effect such as in [Gammaitoni et al., 2011] and [Andò et al., 2010]. Figure 10(b) shows the numerically computed harvested power from the PWL system using the following expression 
for a sufficiently large value of $N$.

$$
P_{\mathrm{avg}}=\frac{1}{N T} \int_{0}^{N T} \beta_{e}(x) y^{2}(t) \mathrm{d} t
$$

Figure 11(b) shows the average harvested power from the PWL system in terms of the force intensity for different values of driving force $\omega_{f}$. For low values of the forcing amplitude the average power increases proportionally to the force intensity. However, it can also be noticed a sudden jump of the amplitude at a certain critical value of force intensity which is depending on the driving frequency. This behavior has been also observed in [Triplett et al., 2011]. Note however that this will occur only if the driving frequency is selected within an appropriate interval as it can be observed in Fig. 10(b).

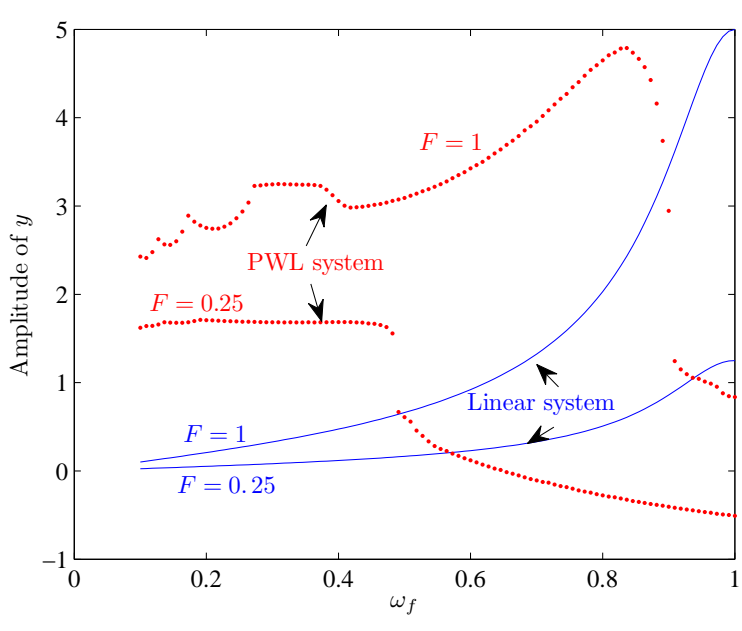

(a)

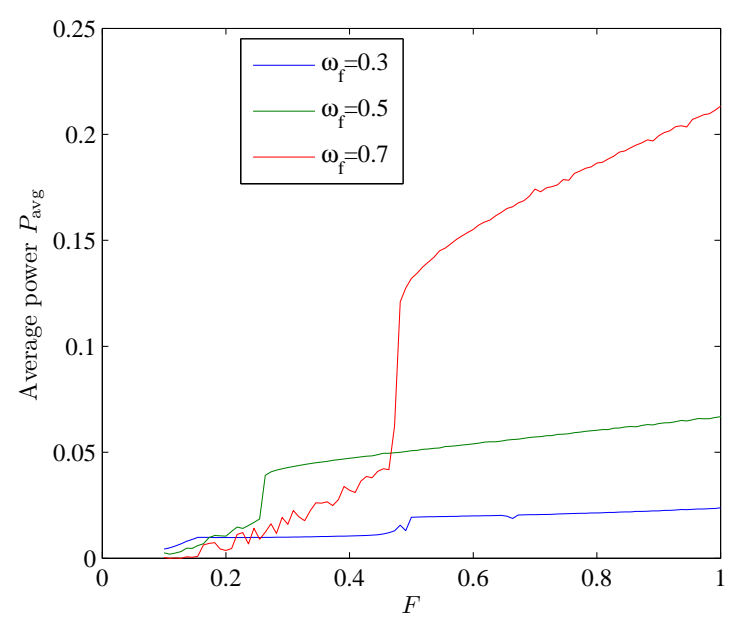

(b)

Fig. 11. Frequency responses of the PWL system and linear equivalent system for different values of $F$ (a) and the average harvested power of the PWL system in terms of the force intensity $F$ for different values of driving frequency $\omega_{f}(b)$.

\subsubsection{Noisy excitation}

In this case, a band limited white noise is added to the harmonic excitation force of the system. The maximum bandwidth $\omega_{\mathrm{bw}}$ and the noise power $P_{n}$ are two parameters that merit special attention but we have fixed them to $\omega_{\mathrm{bw}}=5 \sqrt{k / m}$ and $P_{n}=P_{s} / 10$, where $P_{s}=F^{2} / 2$ is the sinusoidal force power. As before, case study 2 is considered corresponding to $x_{e}=0.5, b_{m}=0.2$ and $b_{e}=0.4$. Figure 12 shows the time domain response and the trajectory of the system for $F=0.45$. Comparing this figure with Figs. 6(a) and 7(a), it can be observed that the existence of a noisy environment does not alter significantly the dynamics of the system. A mesh plot of the average harvested power is depicted in Fig. 13(a). Compared with Fig. 10(b), the performance of the PWL system with a noisy excitation, in terms of the harvested power, is similar to the one with a purely harmonic vibration source. The efficiency $\eta$ of the system defined as the ratio between $P_{\text {avg }}$ the average harvested power and the total average excitation power $P_{\text {tot }}$ [Hendijanizadeh et al., 2013] is also computed and it is shown in Fig. 13(b). It has been observed that the region of maximum efficiency do not correspond always to the region of the maximum harvested power and that the efficiency $\eta$ in this region can reach values between $50 \%$ and $60 \%$.

\section{Conclusions and discussions}

In this work, we studied the dynamics of a PWL spring-mass single degree of freedom system for energy harvesting applications. The linear configurations corresponding to different regions of the state-space, where stiffness 


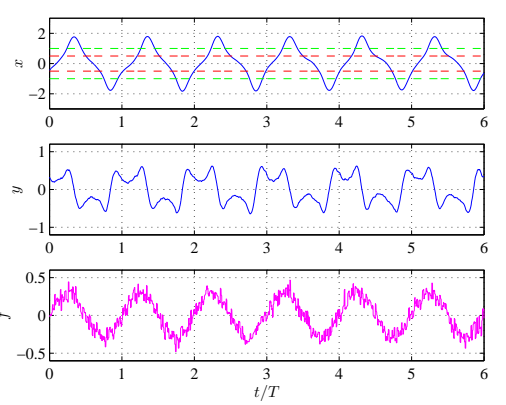

(a)

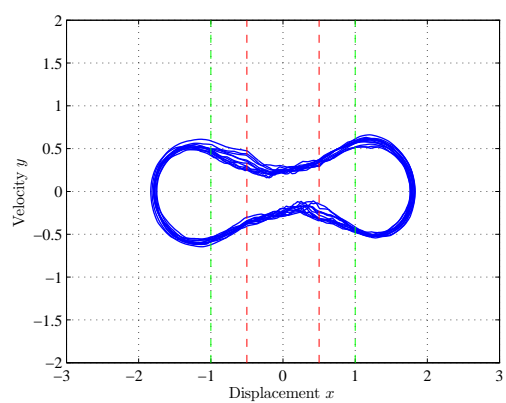

(b)

Fig. 12. Time-domain waveforms (a) and state-space trajectory (b) for $F=0.45$.

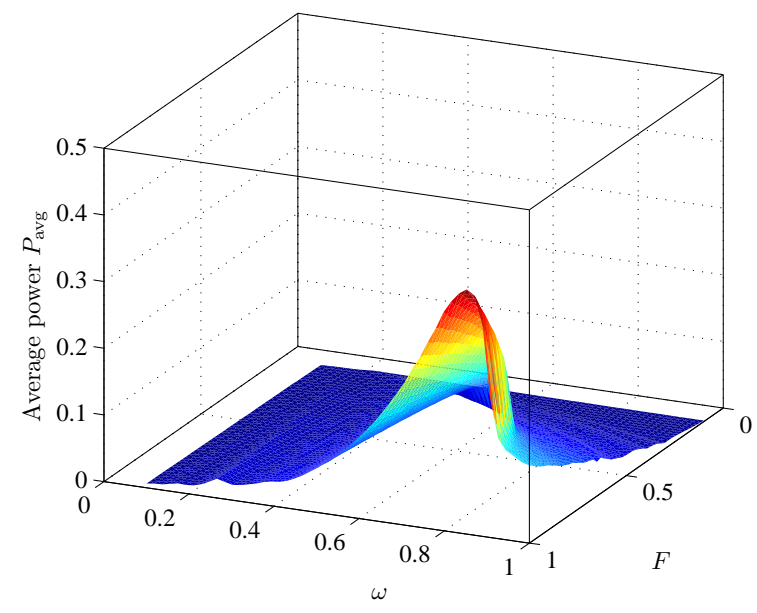

(a)

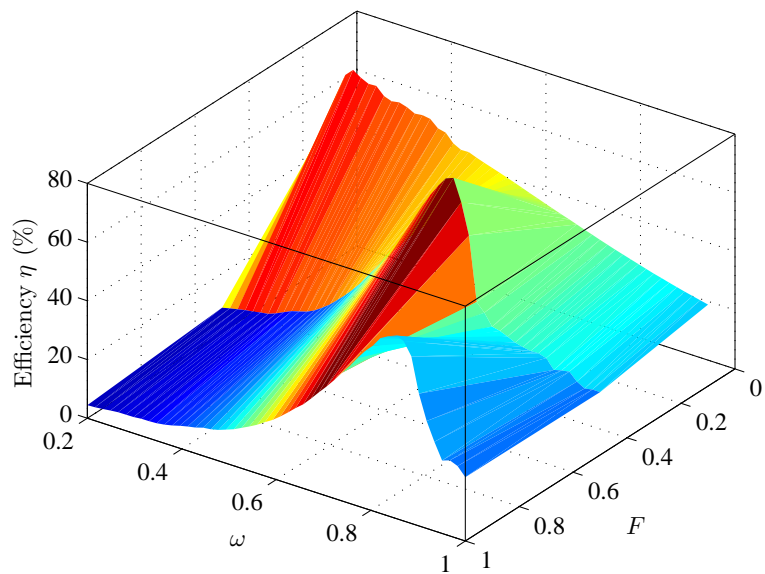

(b)

Fig. 13. Numerically obtained average harvested power of the PWL system and its efficiency for different values of the force intensity $F$ and bandwidth $\omega_{\mathrm{bw}}$ of the random excitation.

and restoring force take different values, were derived. The frequency response and time-domain waveforms were obtained and compared in this study. It was shown through long-time integration of the dynamical model and the corresponding frequency response, that the harvested power could be larger than that obtained from a linear equivalent system for both purely harmonic and noisy vibrational excitations. In particular, we found that for relatively low frequencies and intensities of the vibration source, the average harvested energy in the PWL system is larger as compared to the linear equivalent system. This could be an advantage for potential use of this system in low frequency ambient vibrational-based energy harvesting. The numerical results show that the system exhibits a rich variety of nonlinear phenomena such as pitchfork and flip bifurcations, coexistence of solutions and chaotic behavior. Stability analysis of the PWL system was performed using three different methods taking a special care with its switching nature and a good matching between them was obtained. It is worth noting that the cases which it has been possible to describe in the present paper are very limited. This can be ascribed quite simply to the fact that many other switching sequences, not considered in this study, can also take place for certain sets of parameter values. However, the analysis can be drastically simplified if the border corresponding to the damping is eliminated, as it was done in case study 4 , since it has been verified that dynamics is depending mainly on the effective average total damping. 


\section{Acknowledgments}

The authors would like to thank the anonymous reviewers and the associate editor for many comments and suggestions that has helped to improve an original version of this paper. This work has been partially supported by the Spanish Ministry of Science and Innovation under grant DPI2010-16481.

\section{References}

Aizerman M. A. and Gantmakher F. R. [1958] "On the stability of Periodic Motions", Journal of Applied Mathematics and Mechanics (Translated form Russian) 1(1958).

Andò A., Baglio S., Trigona C., Dumas N., Latorre L. and Nouet P. [2010] "Nonlinear Mechanism in MEMS Devices for Energy Harvesting Applications", Journal of Micromechanics and Microengineering, 20(12): 1-12, 2010.

Bendame M. and Abdel-Rahman E. [2012] "Nonlinear Modeling and Analysis of a Vertical Springless Energy Harvester", MATEC Web of Conferences 1, 01004 (2012), CSNDD 2011, Marrakech, Morocco, DOI: 10.1051/matecconf/20120101004, published by EDP Sciences, 2012.

El Aroudi A., Debbat M. and Martinez-Salamero L. [2007] "Poincaré Maps Modelling and Local Orbital Stability Analysis of Discontinuous Piecewise Affine Periodically Driven Systems", Nonlinear Dynamics, 50(3): 431445, 2007.

El Aroudi A., Ouakad H. and Younis M. [2013-a] "Nonlinear Dynamics of A Piecewise Linear Energy Harvesting System", 1st Euro-Mediterranean Conference on Structural Dynamics and Vibroacoustics 23-25 Apr. 2013, Marrakech (Morocco).

El Aroudi A., Lopez-Suarez M., Alarcon E., Rurali R., Abadal G. [2013-b] "Nonlinear Dynamics in a Graphene Nanostructured Device for Energy Harvesting", IEEE International Symposium on Circuits and Systems (ISCAS'2013), 2727-2730, 19-23 May 2013, Beijin (China).

Filippov A. F. [1988] Differential Equations with Discontinuous Righthand Side, Kluwer Academic Publishers, Dordrecht, 1988.

Gammaitoni L., Vocca H., Neri I., Travasso F. and Orfei F. [2011] "Vibration Energy Harvestng: Linear and Nonlinear Oscillator Approaches," in Sustainable Energy Harvesting Technologies-Past, Present and Future,: 171190, 2011.

Giaouris D., Banerjee S., Zahawi B., Pickert V. [2008] "Stability Analysis of the Continuous-Conduction-Mode Buck Converter Via Filippov's Method”, IEEE Transactions on Circuits and Systems I: Regular Papers, 55(4):10841096, 2008.

Gammaitoni L., Neri I., and Vocca H. [2009] “Nonlinear Oscillators for Vibration Energy Harvesting,” Applied Physics Letters, 94(16): 164102-1/164102-3, 2009.

Hendijanizadeh M., Sharkh S. M., Elliott S. J., and Moshrefi-Torbati M. [2013] "Output power and efficiency of electromagnetic energy harvesting systems with constrained range of motion", Smart Mater. Struct, (22) 125009 (10 pages), 2013.

Kaźmierski T. J. \& Beeby S. (Eds) [2001] "Energy Harvesting Systems. Principles, Modeling and Applications," Springuer, 2011.

Leine R. L. and Nijemeijer H. [2004] Dynamics and Bifurcations of Non-Smooth Mechanical Systems, Lecture Notes in Applied and Computational Mechanics, Springer, 2004.

Liberzon, D. [2003] Switching in Systems and Control, Springuer, 2003.

Liu H. Cho J. T., Chenggen Q., Kobayashi T., Chengkuo L. [2011] "Piezoelectric MEMS Energy Harvester for Low-Frequency Vibrations With Wideband Operation Range and Steadily Increased Output Power," Journal of Microelectromechanical Systems, 20(5):1131-1142, 2011.

López-Suárez M., Rurali R., Gammaitoni L. and Abadal G. [2011] "Nanostructured Graphene for Energy Harvesting," Physical Rweview B, 84(16):1-5, 2011.

Mandal K., Banerjee S., and Chakraborty C. [2013-a] "Symmetry-Breaking Bifurcation in Series-Parallel Load Resonant DC-DC Converters," IEEE Transactions on Circuits and Systems-I, 60(3): 778-787, 2013.

Mandal K., Chakraborty C., Abusorrah A., Al-Hindawi M., Al-Turki Y. and Banerjee S. [2013-b] "An Automated Algorithm for Stability Analysis of Hybrid Dynamical Systems," The European Physical Journal, Special Topics (222):757-768, 2013.

Mandal K. and Banerjee S. [Mandal and Banerjee 2012] "A Software for Stability Analysis of Switching Electronic 
Circuits," National Conference on Nonlinear Systems and Dynamics (NCNSD), Pune, India, July 12-15, 2012. Massana R. and Daqaq F. [2012] "Energy Harvesting in the Super-harmonic Frequency Region of a Twin-well Oscillator," Journal of Applied Physics, 111(4):0445011, 1-11, 2012.

Mitcheson P. D, Yeatman E. M., Rao G. K, Holmes A. S. and Green T. C. [2008] "Energy Harvesting From Human and Machine Motion for Wireless Electronic Devices", Proceedings of the IEEE, 96(9), 1457-1586, 2008.

Nayfeh A.H., Ouakad H. M., Najar F., Choura S. and Abdel-Rahman E. [2009] "Nonlinear Dynamics of a Resonant Gas Sensor," Nonlinear dynamics, 59(4): 607-618, 2009.

Nayfeh A. H., and Balachandran B. [1995] “Applied Nonlinear Dynamics,” Wiley, New York, 1995.

Ralman R., Brennan M. J., Mace B. R. and Kovacic I. [2010] "Potential Benefits of a Non-linear Stiffness in an Energy Harvesting Device,” Nonlinear Dynamics, 59(4):545-558, 2010.

Soliman M. S. M., Abdel-Rahman E. M., El-Saadany E. F. and Mansour R. R. [2008] "A Wideband Vibration-based Energy Harvester," J. Micromech. Microeng., 18, 115021: 1-11), 2008.

Stephen N.G. [2011] "On Energy Harvesting From Ambient Vibration," Journal of Sound and Vibration, 293(12):409-425, May 2006.

Tang L., Yang Y. and Soh K. [2000] “Toward Broaband Vibration-based Energy Harvesting," Journal of Intelligent Material Systems and Structures, 21: 1867-1896, 2010.

Toh T. T., Bansal A., Hong G., Mitcheson P. D, Holmes A. S and Yeatman E. M [2007] "Energy Harvesting from Rotating Machines", Proc. PowerMEMS, Freiburg, Germany, 327-330, 2007.

Trigona C., Dumas N., Latorre L., Andò B., Baglio S. and Nouet P. [2011] "Exploiting Benfits of a PeriodicallyForced Nonlinear Oscillator for Energy Harvesting from Ambient Vibration," Procedia Engineering: 25:, 819822, 2011.

Triplett A., Linton J. and Quinn D. D. [2011] “Electromagnetic Energy Harvesting,” ENOC 2011, 24-29 July 2011, Rome, Italy.

Tulloch R. [2011] “Investigating Non-linear Vibrations for Energy Harvesting,” Master of Engineering, University of Bristol, 2011.

Xie F, Zhang B., Yang R., H.-C. Iu [2013] "Detecting Bifurcation Types and Characterizing Stability in DC-DC Switching Converters by Duplicate Symbolic Sequence and Weight Complexity," IEEE Transactions on Industrial Electronics, 60(8): 3145-3156, 2013.

Xiong X., Tse C. K. and Ruan X. [2013] "Smooth and Non-Smooth Bifurcations in Multi-Structure Multi-OperatingMode Hybrid Power Systems," International Journal of Bifurcation and Chaos, 23(5) 1350094: 1-12, 2013. 